\title{
Erratum: Fractional quantum Hall effect in CdTe [Phys. Rev. B 82, 081307(R) (2010)]
}

B. A. Piot, J. Kunc, M. Potemski, D. K. Maude, C. Betthausen, A. Vogl, D. Weiss, G. Karczewski, and T. Wojtowicz (Received 9 December 2013; published 18 December 2013)

An error of a factor of 2 is displayed on the $x$ axis of Fig. 2(d): It should read $B_{\text {total }}=2 \times 5=10 \mathrm{~T}$ instead of $B_{\text {total }}=5 \mathrm{~T}$. In the main text, page 3, paragraph 5 , the sentence "For $v=4 / 3\left(v_{\mathrm{CF}}^{*}=2\right)$, these crossings occur for $B_{\text {total }} \sim 3.4 \mathrm{~T}$ and $B_{\text {total }} \sim 6.8 \mathrm{~T}$, respectively," should be replaced with "For $v=4 / 3\left(v_{\mathrm{CF}}^{*}=2\right)$, these crossings occur for $B_{\text {total }} \sim 6.8 \mathrm{~T}$ and $B_{\text {total }} \sim 13.6 \mathrm{~T}$, respectively...."

Also, the sentence "The same conclusions are drawn for the $v=5 / 3\left(v_{\mathrm{CF}}^{*}=1\right) \mathrm{FQH}$ state, provided $B_{\text {total }}>3$ T" should be replaced with "The same conclusions are drawn for the $v=5 / 3\left(v_{\mathrm{CF}}^{*}=1\right) \mathrm{FQH}$ state, provided $B_{\text {total }}>6$ T."

None of the interpretations and conclusions of the paper are affected by these errors. 